\title{
A detecção precoce dos fatores de risco relacionados à prematuridade e suas implicações para a Educação Especial
}

\author{
Luciana Pizzani* \\ Juliana Lopes** \\ Claudia Maria Simões Martinez $z^{* * *}$
}

\section{Resumo}

Diversos fatores podem comprometer o crescimento e o desenvolvimento dos indivíduos, dentre eles encontra-se a prematuridade e o baixo peso ao nascimento. Sendo assim, este artigo tem como objetivo descrever a evolução dos conceitos relacionados à prematuridade e aos fatores de riscos que levam a sua ocorrência. Nesse contexto, são enfatizadas também, as medidas de prevenção e intervenção precoce nas áreas da Educação, Educação Especial e Saúde que podem contribuir sobremaneira para o completo desenvolvimento dos indivíduos nascidos prematuramente.

Palavras-chave: Prematuridade; Prevenção; Educação Especial.

\footnotetext{
* Doutoranda do Programa de Pós-Graduação em Educação Especial da Universidade Federal de São Carlos (UFSCar), São Carlos, São Paulo, Brasil.

** Mestranda do Programa de Pós-Graduação em Terapia Ocupacional da Universidade Federal de São Carlos (UFSCar), São Carlos, São Paulo, Brasil.

*** Professora Doutora do Programa de Pós-Graduação em Terapia Ocupacional da Universidade Federal de São Carlos (UFSCar), São Carlos, São Paulo, Brasil.
} 


\section{Early detection of risk factors related to prematurity and this implications for Special Education}

\section{Abstract}

Several factors can compromise growth and development of individuals, among them are prematurity and low birth weight. Therefore, this article aims to describe the evolution of concepts related to prematurity and risk factors that lead to its occurrence. In this context, are also emphasized, measures of prevention and early intervention in the areas of Education, Special Education and Health can contribute greatly to the full development of individuals born prematurely.

Keywords: Prematury; Prevention; Special Education.

\section{Introdução}

A prematuridade pode ser um dos diversos fatores de risco que podem comprometer o crescimento e o desenvolvimento dos indivíduos. Segundo Vaz (1986), é um dos grandes problemas de saúde pública e muito contribui para a elevação do número de casos de morbi-mortalidade infantil e também para a elevação do número de casos de invalidez, principalmente em países em desenvolvimento.

A presença de fatores de risco é uma constante na vida do homem, não somente em sua concepção, mas também durante todo seu desenvolvimento. Tais fatores podem estar presentes antes, durante e depois do nascimento podendo resultar em condições prejudiciais ao desenvolvimento do bebê (RODRIGUES, 2003).

Nesse sentido, o objetivo deste artigo é relacionar alguns aspectos da prematuridade, descrevendo a evolução dos seus conceitos e os fatores de risco que levam a sua ocorrência.

Também são enfatizadas as medidas de prevenção e intervenção precoce nas áreas da Educação, Educação Especial e Saúde que podem contribuir sobremaneira para o completo desenvolvimento dos indivíduos nascidos prematuramente. 
A evolução dos conceitos e das técnicas relacionadas ao diagnóstico da prematuridade

Realizada em Nova lorque em 1948, a primeira Assembleia Mundial de Saúde reconheceu a importância da prematuridade como a grande causa universal de mortalidade infantil e adotou para ela uma definição internacional: o prematuro é o neonato cujo peso de nascimento é $2500 \mathrm{~g}$ ou menos. O Comitê da Organização Mundial de Saúde, especializado em prematuridade, adotou essa definição internacional, mas percebeu que não seria aplicável em todos os países. Em muitas partes do mundo, ela foi útil para diferenciar os recém-nascidos que requeriam certos cuidados especiais; mas, em outros países, o uso desse padrão fez elevar em grandes proporções o número de recém-nascidos considerados prematuros. Muitos dos quais, segundo a antiga pesagem admitida, não tinham nascido prematuramente e não pareciam necessitar de cuidados especiais. Esse fato levou à adoção local de diversos níveis de baixo peso de nascimento, gerando confusão e impedindo comparações (CROSSNE, 1980).

Houve necessidade de uma reavaliação. Um estudo da Organização Mundial de Saúde sobre o peso de nascimento foi realizado em 18 países em diversos estágios de desenvolvimento socioeconômico. Esse estudo mostrou que a proporção indevidamente aumentada de crianças com peso de nascimento de $2500 \mathrm{~g}$ ou menos não era devida a um aumento da proporção de nascidos prematuramente (antes de completar 37 semanas de gestação), mas a uma redução geral do peso de nascimento em todas as fases de maturação. Muitos dos recém-nascidos, que pesaram $2500 \mathrm{~g}$ ou menos ao nascerem, tinham idade gestacional maior do que 37 semanas, mas possuíam baixo peso. Em vista da evidência convincente de que muitos desses neonatos incluídos na definição internacional não nasceram prematuramente, o Comitê Especializado em Saúde Materno-Infantil da Organização Mundial de Saúde em 1961 recomendou que o conceito de prematuridade deveria ser substituído pelo de baixo peso de nascimento (CROSSNE, 1980).

Sendo assim, a Organização Mundial de Saúde definiu o conceito de prematuridade como o nascimento abaixo de 37 semanas gestacionais, e atualmente é a prematuridade, assim considerada, a principal causa de morte, morbidade e incapacidade infantil no mundo (WHO, 2010).

Segundo a Organização Mundial de Saúde, a definição da duração de gestação a termo, que também é adotada pelo Comitê de Neonatologia da Sociedade Brasileira de Pediatria, é de 37 semanas completas (259 dias). Por sua vez, o Comitê de Feto e Recém-Nascido da Academia Americana de Pediatria estabelece-a como aquela que ocorre no período de 38 semanas, 
pois considera que a morbimortalidade de neonatos entre 37 e 38 semanas difere da apresentada pelos nascidos com 38 ou mais semanas de gestação. Considera-se, então, que o recém-nascido a termo tem o nascimento entre 38 e 42 semanas e o pós-termo após 42 semanas completas (GONÇALVES; JORGE, 2006).

A definição da idade gestacional do recém-nascido pode ser realizada por diversos métodos nos períodos pré e pós-natal.

O método utilizado para a determinação no período pré-natal, comumente encontrado na literatura, é o cálculo por meio das semanas da amenorreia, conhecida como a regra de Nägele, que se baseia na data inicial do último período menstrual (DUM) e que é praticada desde 1833. Essa regra consiste em adicionar 7 dias e 9 meses à DUM (ou diminuir 3 meses, quando se faz o cálculo retrógrado). É ainda o método isolado mais importante para calcular a data provável de partos a termo, porém esbarra na dificuldade de obter a DUM com precisão, devido a eventuais ciclos menstruais irregulares, sangramentos pós concepcionais e uso de contraceptivos (GONÇALVES; JORGE, 2006).

Outras técnicas citadas por Gonçalves e Jorge (2006) são: a) a medida da altura do fundo uterino, que pode ser induzida a erros devido a gestações múltiplas, retardo ou aumento patológico do crescimento fetal; b) o estudo do líquido amniótico; c) a ultrassonografia fetal e obstétrica, que fornecem indicadores da maturidade orgânica do feto, em especial sua maturidade pulmonar, por meio da detecção dos fosfolípedes componentes do surfactante pulmonar fetal, cujas idades de aparecimento e concentrações no líquido amniótico estão bem estabelecidas.

Com os avanços da tecnologia, a ultrassonografia fetal e obstétrica colocam esse método como o mais confiável para obter a idade gestacional de um concepto, segundo Gonçalves e Jorge (2006). Deve ser realizado entre a $15^{a}$ e $19^{a}$ semana de gestação, quando o erro de avaliação é de apenas 9 dias. Ultrapassado esse período, a margem de erro aumenta com o evoluir da gravidez, em decorrência de fatores materno-placentários que podem influir significativamente sobre o crescimento do feto, retardando-o ou acelerando-o.

Os métodos desenvolvidos após o nascimento utilizam critérios somáticos e neurológicos para a determinação da idade gestacional nas primeiras horas de vida.

Segundo Ohlweiler (2001), as técnicas de avaliação neurológica decorreram naturalmente dos estudos da escola francesa sobre exame 
neurológico de prematuros, dado o caráter evolutivo dos achados neurológicos. Os trabalhos de Saint-Anne Dargassies (1954, 1955, 1966), Thomas et al. (1960), Farr et al. (1966) e Amiel-Tison (1968) foram grandes contribuintes para o desenvolvimento de um escore de avaliação neurológica para a determinação da idade gestacional. Lubchenco (1970) publicou uma tabela para avaliação da idade gestacional com base no exame físico e neurológico do RN.

Gonçalves e Jorge (2006) relatam que foi a partir de 1970 que os métodos foram se tornando mais precisos. Naquele ano, o médico pediatra Dubowitz desenvolveu um método de avaliação da idade gestacional - 0 chamado Método de Dubowitz. Essa avaliação acontece apoiada em 21 itens somáticos e neurológicos. Esses itens são divididos em 11 sinais físicos (edema, textura da pele, cor da pele, opacidade da pele, lanugem, pregas plantares, formação de mamilo, tamanho do nódulo mamário, forma da orelha, firmeza da orelha, genitália masculina ou genitália feminina) e 10 sinais neurológicos (postura, ângulo do punho, dorsiflexão do tornozelo, recolher do braço, recolher da perna, ângulo poplíteo, manobra calcanharorelha, sinal de cachecol, queda da cabeça, suspensão ventral). É um método preciso, porém não é o mais utilizado pelos neonatologistas devido à grande quantidade de itens a serem avaliados tornando assim a avaliação extensa.

Em 1978, a partir do método de Dubowitz, surge o Método de Capurro. Haroldo Capurro e seus colaboradores, em um trabalho publicado em 1978 intitulado Um método simplificado para o diagnóstico da idade gestacional em criança recém-nascida, comentam que as vantagens do método de Dubowitz eram o fato de ele ser indolor, de baixo custo, preciso e permissor da quantificação da idade gestacional a partir do exame clínico do neonato. Entretanto, tornava-se complexo para a prática diária, devido ao elevado número de variáveis a serem consideradas. Propuseram, então, o Método de Capurro, que se subdivide em dois métodos: o somático e o somatoneurológico (GONÇALVES; JORGE, 2006).

O primeiro é utilizado quando os bebês apresentam algum dano cerebral, ou disfunção neurológica observada no nascimento e traduz-se em um somatório entre uma constante de 204 dias e a nota obtida na avaliação de cinco itens somáticos (textura da pele, forma da orelha, nódulo mamário, pregas plantares e formação do mamilo). O segundo método utilizado, em caso de bebês que não apresentaram alteração neurológica no nascimento, é obtido por meio de um somatório de pontos que se referem aos quatro itens somáticos (quatro primeiros citados anteriormente) e dois neurológicos (sinal de cachecol e posição da cabeça: levantando o recém-nascido) a uma constante de 200 dias (CAPURRO et al., 1978 apud CARVALHO, 2005). 
Com a finalidade de ampliar a precisão das idades gestacionais e incluir tanto recém-nascidos mais prematuros quanto os mais maduros, foi criado em 1991 o novo método de Ballard-New Ballard Score, em modificação ao teste original desenvolvido em 1979. O teste abrange o período de 20 a 44 semanas de idade gestacional, sendo constituído por seis itens de maturidade neuromuscular (postura, angulação do punho, recuo do braço, ângulo poplíteo, sinal de cachecol e calcanhar à orelha) e seis itens de maturidade física (pele, lanugem, superfície plantar, tecido mamário, olho e orelha, genitais masculino ou femininos). A idade gestacional em semanas é obtida por meio do somatório de pontos de avaliação dos itens (BALLARDI, 1991 apud CARVALHO, 2005).

A determinação da idade gestacional, segundo Ramos et al. (2002), tem como finalidade determinar o grau de maturidade do recém-nascido. Para isso, os neonatologistas se baseiam em dados selecionados pelo exame físico e neurológico. Ou seja, baseiam-se em um conjunto de tonos e reflexos neurológicos que, usados criteriosamente, permitem avaliar a idade do RN independentemente do crescimento fetal, com aproximação de até uma a duas semanas. Com o passar do tempo, vários métodos foram elaborados, porém observa-se que o Método Capurro tem sido mais utilizado, pois permite aplicação mais simples e atende às necessidades dos serviços.

A prematuridade pode ser classificada em prematuridade limítrofe, moderada e extrema de acordo com os problemas clínicos característicos de cada fase gestacional (LEONE; RAMOS; VAZ, 2002).

A prematuridade limítrofe compreende o período de gestação de 35 a 36 semanas, com crianças pesando entre 2200 e $2800 \mathrm{~g}$, medindo entre 45 e $46 \mathrm{~cm}$ de comprimento e tendo aproximadamente $32,5 \mathrm{~cm}$ de perímetro cefálico. Respondem por $7 \%$ de todos os nascimentos vivos, por $65 \%$ dos recém-nascidos pré-termo nascidos vivos. Dentro desse tipo de prematuridade, a mortalidade é muito baixa, cerca de 0,9\% (LEONE; RAMOS; VAZ, 2002).

A prematuridade moderada varia de 31 a 34 semanas gestacionais com peso superior a $2000 \mathrm{~g}$. Em centros mais desenvolvidos, prematuros dentro de tais condições apresentam baixa mortalidade, sendo os casos de membrana hialina grave o maior responsável pelos óbitos. Já nos centros menos desenvolvidos, as infecções são os eventos mais importantes nesse grupo (LEONE; RAMOS; VAZ, 2002).

A prematuridade extrema compreende gravidez inferior a 30 semanas e os recém-nascidos costumam pesar menos de $1500 \mathrm{~g}$, medir menos que 38 $\mathrm{cm}$ de estatura e menos que $29 \mathrm{~cm}$ de perímetro encefálico ao nascerem. 
Em decorrência dessa maior imaturidade, apresentam intercorrências mais graves e frequentes, favorecendo o aparecimento de deficiências a curto e/ ou longo prazo (LEONE; RAMOS; VAZ, 2002).

Essas intercorrencias podem ser: asfixia perinatal, dificuldade na manutenção de temperatura corpórea, insuficiência respiratória (doença de membranas hialinas, displasia broncopulmonar, crises de apneia, hiperbilirrubinemia, infecções adquiridas, hipo e hiperglicemia, hipocalcemia precoce, enterocolite necrosante, hemorragia intracraniana, persistência de canal arterial (PCA), retinopatia da prematuridade, doença metabólica óssea, anemia, malformações congênitas, latrogenias), infusão de líquidos e eletrólitos, efeitos adversos de drogas (LEONE; RAMOS; VAZ, 2002).

Quanto ao peso no momento do nascimento, Trindade e Lyra (2006) apontam três subdivisões: 1) prematuros de baixo peso: recém-nascidos com peso igual ou inferior a $2500 \mathrm{~g} ; 2$ ) prematuros de muito baixo peso: com peso igual ou inferior a $1500 \mathrm{~g}$; 3 ) prematuros com extremo baixo peso: peso igual ou inferior a $1000 \mathrm{~g}$.

Torna-se fundamental conhecer o peso de nascimento e a idade gestacional para que se possa fazer uma boa avaliação das condições de vitalidade do recém-nascido. Por isso, em 1967, Battaglia e Lubchenco propuseram uma classificação dos recém-nascidos vivos de 24 a 42 semanas baseados na curva de crescimento intra-uterino entre os percentis 10, 25, 50, 75 e 90, caracterizando os recém-nascidos em três grupos segundo o peso: adequado para idade gestacional (AIG) com peso entre percentis 10 e 90; pequeno para a idade gestacional (PIG) com peso abaixo do percentil 10; grande para a idade gestacional (GIG) com peso acima do percentil 90 (RAMOS et al., 2002).

A prematuridade é a principal causa de morbidade e mortalidade neonatais, e sua incidência varia de acordo com as características da população analisada. A prevenção é um dos grandes desafios, pois não se trata apenas de um problema de ordem médica, mas educativo e social, o que o torna mais complexo. Portanto, fatores de risco sociais e biológicos podem levar à prematuridade (VAZ, 1986; BITTAR; ZUGAIB, 2002).

Esses fatores sociais e biológicos podem ser divididos em seis categorias:

1) Fatores epidemiológicos (sociais): baixo nível socioeconômico, desnutrição, gravidez indesejada, estresse, assistência pré-natal inadequada, fumo, drogas, álcool, idade materna baixa ( $<16$ anos) ou idade superior a 35 anos, estado civil, educação, conflitos familiares, tipo de ocupação da mãe, 
alteração de peso inadequado da mãe, cor da pele, tipo de ocupação da mãe e outros.

2) Obstétricos: infecção amniótica, rotura prematura de membranas, alterações hormonais, sangramentos vaginais de primeiro e segundo trimestres, placenta prévia, deslocamento prematura de placenta, incompetência istmocervical, gemelaridade/poliidrâmnio, malformações fetais e placentárias, partos prematuros anteriores, gestações sucessivas com pequenos intervalos entre os partos, baixo ganho de peso durante a gestação, assistência pré-natal ausente ou tardia, entre outros.

3) Ginecológicos: amputação de colo uterino, malformações uterinas, miomas.

4) Clínico-cirúrgicos: doenças maternas, procedimentos cirúrgicos na gravidez.

5) latrogênicos: a não realização ou demora do exame de ultrassom para determinar a idade gestacional ocorrendo interrupções prematuras da gestação geralmente por cesáreas eletivas.

6) Fatores desconhecidos pelos profissionais ligados à saúde.

É de grande importância conhecer os fatores sociais e biológicos que levam ao trabalho de parto prematuro, pois o seu mecanismo etiológico é desconhecido. A associação desses fatores permitem realizar intervenções terapêuticas que podem contribuir para evitar ou diminuir os prejuízos em longo prazo de crianças nascidas prematuras (VAZ, 1986; IEMMA, 2010).

\section{A detecção precoce dos fatores de risco e suas implicações para a Educação Especial}

A prevenção de qualquer deficiência depende da identificação precoce dos chamados indivíduos de risco. Por meio da rápida identificação dos fatores de risco biológicos, médico-biológicos e multifatoriais e o encaminhamento das crianças para serviços especializados, ocorre a possibilidade da realização de trabalhos preventivos, por meio de programas de promoção de saúde e de intervenção precoce (UFSCar, 2011).

Para Willrich, Azevedo e Fernandes (2009), quanto maior o número de fatores de risco atuantes, maior será a possibilidade do comprometimento do desenvolvimento. Sendo a prematuridade um fator de risco, é fundamental avaliar e intervir com foco na prevenção, a fim de ativar recursos da criança 
e estimular a proteção mediada nos contextos dos ambientes familiar e educacional, e - se possível - no contexto terapêutico. Reafirma-se a importância de planejar programas para auxiliar no desenvolvimento de crianças nascidas em condições de risco, capazes de neutralizar precocemente as alterações presentes no desenvolvimento e comportamento antes do ingresso na fase escolar (LINHARES, 2004).

Conforme Lejarraga (2002), a detecção precoce de problemas no desenvolvimento é importante porque permite ação oportuna com consequente melhor evolução a longo prazo. É necessário ater-se à promoção do desenvolvimento e às medidas de prevenção que favoreçam o desenvolvimento de crianças em risco elegíveis de Educação Especial ou de terapias especializadas (LINHARES, 2004). Segundo Amaral, Tabaquim e Lamônica (2005), crianças que são precocemente identificadas com sinais de anormalidades e/ou desvios, se forem acompanhadas com procedimentos de estimulação, demonstram melhora nas condições de resposta e rearranjo na plasticidade cerebral, capazes de minimizar impacto no desenvolvimento estrutural e funcional, tendo assim uma melhora na qualidade de vida.

Segundo Herren e Herren apud Martins e Moser (1996), a estimulação precoce é um conjunto de processos preventivos e/ou terapêuticos para assegurar à criança um melhor intercâmbio com o meio em que vive durante a primeira infância.

Na visão de Maria-Mengel e Linhares (2007), a identificação eficaz de crianças em risco começa com um exame dos fatores que contribuem para as desordens da criança, tornando-a vulnerável ao enfrentamento das tarefas evolutivas do ciclo vital. Os riscos no desenvolvimento podem estar presentes na própria criança (componentes biológicos, temperamento e a própria sintomatologia), na família (história parental e dinâmica familiar) ou no ambiente (nível socioeconômico, suporte social, escolaridade e contexto cultural).

Scochi et al. (2003) enfatizam que o recém-nascido pré-termo e de baixo peso está sujeito ao duplo risco, social e biológico, podendo ocorrer prejuízos em seu processo de crescimento e desenvolvimento. Parte dos bebês que nascem com fatores de risco necessitam de acompanhamento e de intervenções para garantir seu desenvolvimento pleno e de qualidade. Weiss e Fujinaga (2007) argumentam que a longo prazo essas crianças podem apresentar um aumento de sequelas incapacitantes, de doenças crônicas e neurológicas, além de apresentarem dificuldades de aprendizado e distúrbios cognitivos, de linguagem, visão, audição e comportamentais, dentre outros aspectos. 
Figueiras et al. (2003) relatam que inúmeros trabalhos descritos na literatura mundial têm demonstrado que o melhor rendimento dessas crianças pode ser obtido quando elas recebem algum tipo de intervenção precoce de acordo com suas deficiências. Porém, para que recebam precocemente essas intervenções, é necessário identificar essas crianças e encaminhá-las o mais breve possível a serviços especializados. Em outras palavras, cabenos dizer que é papel do profissional que atua na atenção primária, fazer a vigilância do desenvolvimento de todas as crianças, identificar aquelas com necessidades especiais e encaminhá-las oportunamente para tratamento.

Segundo orientações do Ministério da Saúde, os bebês de risco têm o direito de usufruir dos processos de avaliação e intervenção no que se refere às suas necessidades específicas. Esse processo deve analisar os fatores de risco presentes, sobretudo os de caráter ambiental que possam ser removidos ou atenuados, uma vez que podem com grande probabilidade prejudicar o desenvolvimento infantil. Assim, passou-se a dar uma maior atenção à questão do ambiente em que a criança está inserida como fator de grande contribuição para o seu desenvolvimento (BRASIL, 2000).

Para Nunes (1993), a constatação da presença de fatores de risco (biológicos, psicossociais ou genéticos) e consequentemente da identificação das crianças de risco podem ser realizadas antes, durante e após o nascimento.

Os fatores de risco biológicos têm diversas origens. Variam desde anomalias cromossomiais, erros inatos do metabolismo, passando por infecções viróticas, desnutrição da mãe, exposição a elementos químicos, entre outros (NUNES, 1993).

Os fatores psicossociais envolvem variáveis demográficas e processuais. Entre as variáveis demográficas, destacam-se ocupação dos pais, baixo nível intelectual e de escolaridade da mãe, doença mental crônica da mãe, ordem de nascimento da criança, tamanho da família e desorganização familiar. Entre as processuais estão: rigidez das atitudes, crenças e valores da mãe quanto ao desenvolvimento da criança, ansiedade da mãe, organização inadequada do ambiente físico do lar, redução das interações afetivas positivas da mãe com a criança durante a primeira infância (NUNES, 1993).

Estudos como os de Fraga et al. (2008) e Linhares et al. (2003) verificaram a relação entre ansiedade materna e os indicadores de desenvolvimento de crianças nascidas pré-termo. Ficou constatado que o nível de ansiedade materna mais elevado associou-se com um melhor desempenho dos bebês em tarefas cognitivas e um pior em tarefas de 
motricidade ampla e permanência de objetivos. Dessa forma, a ansiedade materna pode atuar como fator de risco ao desenvolvimento motor amplo e como mecanismo de proteção ao desenvolvimento cognitivo.

Nunes (1993) salienta que há, ao longo do tempo, uma contínua interação entre fatores biológicos e ambientais no desenvolvimento da criança. Essas condições tanto podem atenuar quanto agravar os efeitos dos fatores biológicos de risco. Assim, a prevenção depende da identificação precoce dos chamados indivíduos de risco.

\section{Para Perez-Ramos (1992, p. 7)}

A prevenção constitui um ato ou efeito de evitação. Implica em ações antecipadas destinadas a impedir a ocorrência de fatos ou fenômenos prejudiciais à vida e à saúde e, no caso da ocorrência desses fatores e fenômenos, evitar a progressão de seus efeitos.

Segundo Formiga e Pedrazzani (2004, p. 107)

... prevenir as deficiências implica em realizar ações destinadas a impedir a ocorrência de fatos e fenômenos prejudiciais à vida e à saúde e, no caso da ocorrência desses, evitar a progressão de seus efeitos. As ações preventivas dos atrasos e distúrbios do desenvolvimento podem ser conduzidas em três níveis: primário, secundário e terciário.

Muitas crianças chegam tardiamente aos serviços de saúde especializados, apresentando consequentemente algumas sequelas. Para que isso não aconteça, é necessário fornecer informações sobre os serviços de atenção primários, secundários e terciários para a detecção e prevenção das deficiências.

$\mathrm{Na}$ prevenção primária, o objetivo da intervenção é reduzir a incidência de determinadas condições de excepcionalidade na população por meio de identificação, remoção ou redução dos efeitos de fatores de risco que produzem tais condições. Ela tem um caráter educativo e pretende promover melhorias nas condições de saúde, educação, trabalho e moradia para toda a população, principalmente para os segmentos da população considerados mais vulneráveis. São consideradas ações primárias: programas educativos e desenvolvimento e de controle do meio ambiente (antipoluição), instalação de centros de diagnóstico precoce, serviços para crianças adotivas e lares substitutos, programas educacionais para menores (creches e pré-escolas), entre outras (MARTINEZ et al., 2007; FORMIGA; PEDRAZZANI, 2004). 
Para Batista e Enumo (1996), as ações de prevenção podem produzir dois importantes efeitos: 1) redução de gastos com programas de recuperação; 2) redução de sofrimento para o indivíduo bem como para as pessoas que o cercam. Essas ações promovem melhores condições de desenvolvimento biopsicossocial do indivíduo, no período pré-natal (durante a gravidez), perinatal (no momento do parto) e pós-natal (após o nascimento), além de significar medidas de proteção contra agentes causadores de deficiências.

O comportamento preventivo deve ter início no momento em que a mulher decidir ter filhos, ou seja, no período pré-natal, que - segundo Leal e Conrado (2011) - "é um trabalho conjunto da grávida, dos serviços de saúde e da família e destina-se a promover uma boa gestação, criar melhores condições para a realização do parto e proteger a saúde do bebê e da mãe". Para isso, é importante que os serviços de saúde sejam organizados para melhorar o acesso e a qualidade do atendimento pré-natal e a humanização do parto.

No período perinatal, os agentes potencialmente causadores de deficiências podem ser identificados como os problemas ocorridos durante o parto e os cuidados inadequados com recém-nascido. No período neonatal, podem ser apontadas as infecções no berçário, falta de cuidados e de condições hospitalares. No período pós-natal, podem ser apontadas as doenças que aparecem nas crianças como meningite, encefalite, infecções dos órgãos sensoriais, entre outros (BATISTA; ENUMO, 1996, p.5).

Atualmente alguns exames ajudam a detectar a ocorrência de alterações no desenvolvimento fetal, como o vilo corial, a amniocentese, a cardocentese, a ecocardiografia fetal e o Doppler (LEAL; CONRADO, 2011).

Outras medidas preventivas devem ser tomadas para evitar - aparecimento de doenças na mãe como sífilis, rubéola, catapora, toxoplasmose; e para diminuir a exposição da mãe a fatores ambientais como raixo $X$, radioterapia, uso de medicamentos, traumatismos, tentativas de aborto, desnutrição materna, gravidez de alto risco, ingestão de drogas, entre outros (LEAL; CONRADO, 2011; BATISTA; ENUMO, 1996).

Portanto, na prevenção primária, é possível identificar alguns riscos de problemas com o bebê, abrangendo desde as causas hereditárias até algumas deficiências que podem ser provocadas por problemas de saúde da futura gestante (LEAL; CONRADO, 2011).

A prevenção secundária é aquela que tem lugar após se constatar que as condições de excepcionalidade já se instalaram. Essa prevenção 
tem o objetivo de reduzir sua duração e/ou gravidade. Exemplos de ações preventivas secundárias são as desenvolvidas pelos centros de diagnósticos e tratamento das crianças de alto risco e risco comprovado, programas de educação da comunidade e a formação de recursos humanos para atuar com população em creches e pré-escola (MARTINEZ et al., 2007; FORMIGA; PEDRAZZANI, 2004).

Batista e Enumo (1996) também enfatizam diversas medidas que abrangem a prevenção secundária, como: tratamento da sífilis e toxoplasmose, quando instaladas na mãe; pronto atendimento a problemas detectados no parto, como anoxia neonatal, baixo peso de nascimento, dificuldades respiratórias; triagem em massa em berçários para erros inatos do metabolismo; avaliação neurológica do recém-nascido; programas de detecção precoce e triagem populacional para identificação de crianças em risco; pronto atendimento a doenças do bebê e da criança, visando obter a cura ou a menor sequela possível; atendimento por equipe multidisciplinar, o mais precocemente possível de forma a minimizar os danos e a favorecer o seu desenvolvimento.

$\mathrm{Na}$ prevenção terciária, procura-se reduzir as sequelas ou efeitos associados da excepcionalidade por meio de ações que visem minimizar a necessidade de institucionalização, maximizar o potencial de vida independente, reduzir a ocorrência de comportamentos autolesivos esteriotipados e de posturas corporais inadequadas, auxiliar a família a elaborar situações de conflito e de estresse emocional (MARTINEZ et al., 2007; FORMIGA; PEDRAZZANI, 2004).

Batista e Enumo (1996) sugerem algumas iniciativas como a reestruturação do ambiente em termos arquitetônicos e urbanísticos para facilitar o acesso das pessoas com deficiências; disponibilidade de equipamentos para facilitar a locomoção e a comunicação como o sistema Braille, aparelhos para surdez, próteses e órtoses, intérpretes de língua de sinais, programas de TV com legendas e também o aumento de políticas públicas incentivando o respeito às diferenças.

O Ministério de Educação e Cultura (MEC), por meio da Coordenadoria para a Integração da Pessoa Portadora de Deficiência (CORDE), percebendo a importância da prevenção das deficiências e da estimulação precoce dos indivíduos, estabeleceu algumas diretrizes educacionais para essa população. De acordo com a natureza educacional, os programas foram divididos em duas modalidades: unifocal e multifocal. A modalidade unifocal constitui um conjunto sistematizado de ações estimulatórias destinadas a incentivar o desenvolvimento eficaz do processo evolutivo. Já a modalidade multifocal se caracteriza também por ações 
estimulatórias e por sua interligação com as áreas da educação, assistência sócio-familiar, saúde, incluindo a alimentação. Estudos recentes comprovam que esta modalidade demonstrou ser mais benéfica para as crianças submetidas aos programas de estimulação precoce (SANTOS, 2001).

Os atendimentos na modalidade multifocal têm como finalidade proporcionar à criança condições de alcançar seu pleno desenvolvimento, mediante as experiências significativas resultantes de seu contato com pessoas, objetos e espaços; assim como, devem promover mudanças de atitudes das pessoas de seu convívio, particularmente aquelas que assumem a função mediadora no processo como os pais, familiares, educadores, atendentes e outros.

Portanto, pode-se perceber que os profissionais da área de Educação e Saúde estão voltando cada vez mais o enfoque de suas intervenções para a família do bebê pré-termo, considerando a qualidade das interações entre pais/filho e a questão da mediação mãe-bebê, como forma de facilitar as respostas adaptativas da criança no ambiente e também o enfrentamento das situações de risco (FORMIGA; PEDRAZZANI, 2004). Assim, é possível identificar aquelas crianças com necessidades especiais e encaminhá-las oportunamente para os serviços especializados.

\section{Considerações finais}

Sabe-se que os bebês nascidos prematuramente estão sob maior risco de déficit de desenvolvimento e condições de incapacidade que os bebês nascidos a termo. Sendo assim, torna-se relevante conhecer as medidas de prevenção primária, secundária e terciária que contribuem para o desenvolvimento das crianças nascidas prematuramente com a finalidade de intervir precocemente nas possíveis necessidades físicas, emocionais e educacionais, contribuindo sobremaneira para o completo desenvolvimento desses indivíduos.

E, caso seja diagnosticada alguma necessidade específica, a criança deve ser encaminhada o mais breve possível aos serviços especializados, para que a deficiência não se instale; ou, se já instalada, cause o menor dano possível a esse indivíduo. Mais especificamente, na área educacional, a criança deve ser encaminhada aos profissionais da área de Educação Especial que estão habilitados a promover o atendimento e a promoção do desenvolvimento de indivíduos que não se beneficiam de situações tradicionais de educação por limitações ou peculiaridades de diversas naturezas. 


\section{Referências}

AMARAL, A. C. T.; TABAQUIM, M. L. M.; LAMÔNICA, D. A. C. Avaliação das habilidades cognitivas, da comunicação e neuromotoras de crianças com risco de alterações no desenvolvimento. Revista Brasileira de Educação Especial, Marília, v. 11, n. 2, p. 185-200, 2005.

BATISTA, C. G.; ENUMO, S. R. F. Prevenção em saúde - prevenção de deficiências. In: NUNES, L. R. O. P. (Org.). Prevenção e intervenção precoce em Educação Especial. Rio de Janeiro: Associação Nacional de Pesquisa e Pós-Graduação em Psicologia, 1996. p. 1-11.

BITTAR, R. E.; ZIGAIB, M. Parto prematuro: fatores predisponentes e prevenção. In: MARCONDES, E.; VAZ, F. A. C.; RAMOS, J. L. A.; OKAY, Y. Pediatria básica. 9. ed. São Paulo: Sarvier, 2002. v. 1, p. 337-345.

BRASIL. Ministério da Educação. Secretaria de Educação Fundamental. Secretaria de Educação Especial. Referencial curricular nacional para a educação infantil: estratégias e orientações para a educação de crianças com necessidades educacionais especiais. Brasília, 2000. 22 p.

CARVALHO, B. G. E. Triagem visual de bebês prematuros: verificação da aplicabilidade do "método de avaliação da conduta visual de lactentes. 2005. 128 f. Dissertação (Mestrado) - Programa de Pós-Graduação em Educação Especial, Universidade Federal de São Carlos, São Carlos, 2005.

CROSSNE, V. M. O recém-nascido prematuro. 8. ed. São Paulo: Manole, 1980. p. XI-XII.

FIGUEIRAS, A. C. M.; PUCCINI, R. F.; SILVA, E. M. L.; PEDROMÔNICO, M. R. M. Avaliação das práticas e conhecimentos de profissionais da atenção primária à saúde sobre vigilância do desenvolvimento infantil. Caderno de Saúde Pública, São Paulo, v. 6, p. 1691-1699, 2003.

FORMIGA, C. K. M. R.; PEDRAZZANI, E. S. A prevenção de deficiências no alvo da Educação Especial. Revista Brasileira de Educação Especial, Marília, v. 10, n. 1, p. 107-122, 2004.

FRAGA, D. A.; LINHARES, M. B. M.; CARVALHO, A. E. V.; MARTINEZ, F. E. Desenvolvimento de bebês prematuros relacionado a variáveis neonatais e maternas. Psicologia em Estudo, Maringá, v.13, n. 2, p. 335-344, 2008.

GONÇALVES, A. L.; JORGE, S. M. Avaliação da idade gestacional pelo exame clínico do recém-nascido. In: ALVES FILHO, N.; CORREA, M. D.; 
ALVES JUNIOR, J. M. S.; MARCONDES, E.; VAZ, F. A. C.; RAMOS, J. L. A.; OKAY, Y. Pediatria básica. 9. ed. São Paulo: Sarvier, 2002.

IEMMA, E. P. Prematuridade e baixo peso em pré-escolares: fatores de risco ao desenvolvimento da linguagem e alterações fonológico-lexicais. 2010. 98 f. Dissertação (Mestrado) - Programa de Pós-Graduação em Educação Especial, Universidade Federal de São Carlos, São Carlos, 2010.

LEAL, C. S.; CONRADO, L. G. Prevenção um ato de amor - contribuições da Educação para a saúde infantil. Trivium Revista Eletrônica Multidisciplinar, Pitanga-PR, v. 2, n. 1, p. 77-85, 2011.

LEJARRAGA, $\mathrm{H}$. O fascinante processo de desenvolvimento psicomotor da criança. O Berço, Nestlé Nutrition, Alemanha, n. 13, dez. 2002.

LEONE, C. R.; RAMOS, J. L. A.; VAZ, F. A. C. O recém-nascido pré-termo. In: MARCONDES, E.; VAZ, F. A. C.; RAMOS, J. L. A.; OKAY, Y. Pediatria básica. 9. ed. São Paulo: Sarvier, 2002. v. 1, p. 348-352.

LINHARES, M. B. Estresse, resiliência e cuidado no desenvolvimento de neonatos de risco. In: MENDES, E. G. P.; ALMEIDA, M. A.; WILLIANS, L. C. Temas em Educação Especial: avanços recentes. São Carlos: Edufscar, 2004. p. 315-324.

LINHARES, M. B. M.; CARVALHO, A. E. V.; MACHADO, C.; MARTINEZ, F. E. Desenvolvimento de bebês nascidos pré-termo no primeiro ano de vida. Paidéia, Ribeirão Preto, v. 13, n. 25, p. 59-72, 2003.

MARIA-MENGEL, M. R. S.; LINHARES, M. B. M. Fatores de risco para problemas de desenvolvimento infantil. Revista Latino-Americana de Enfermagem, Ribeirão Preto, v. 15, n. esp, p. 837-842, 2007.

MARTINEZ, C. M. S.; JOAQUIM, R. H. V. T.; OLIVEIRA, E. B.; SANTOS, I. C. Suporte informacional como elemento para orientação de pais de pré-termo: um guia para o serviço de acompanhamento do desenvolvimento no primeiro ano de vida. Revista Brasileira de Fisioterapia, Rio de Janeiro, v. 11, n. 1, p. 73-81, 2007.

MARTINS, P. C. R.; MOSER, M. H. Desenvolvimento psicomotor da criança no lar e na creche. Revista Médica Hospital São Vicente de Paulo, [S. I.], v. 8, n. 18 , p. 20-28, 1996.

NUNES, L. R. P. A Educação Especial em creches. Temas em Psicologia, Ribeirão Preto, v. 1, n. 2, p. 109-116, 1993. 
OHLWEILER, L. Desenvolvimento neurológico e resposta apendicular ao movimento do tronco de crianças prematuras, durante o primeiro ano de vida. 2001. 136 f. Tese (Doutorado) - Programa de Pós-Graduação em Medicina, Universidade Federal do Rio Grande do Sul, Porto Alegre, 2001.

PEREZ-RAMOS, A. M. Q. Estimulação precoce: serviços, programas e currículos. Brasília: CORDE, 1992.

RODRIGUES, O. M. P. R. Bebês de risco e sua família: o trabalho preventivo. Temas em Psicologia da SBP, São Paulo, v. 11, n. 2, p. 107-113, 2003.

RAMOS, J. L. A.; CORRADINI, H. B.; VAZ, F. A. C.; BARROS, J. C. R.; NOVO, A. C. C. F. Avaliação da idade gestacional e da adequação do crescimento intra-uterino. In: MARCONDES, E.; VAZ, F. A. C.; RAMOS, J. L. A.; OKAY, Y. Pediatria básica. 9.ed. São Paulo: Sarvier, 2002. v. 1, p. 321-329.

SANTOS, A. P. A. Analise qualitativa de propostas de programas de estimulação precoce. 2001. 13 f. Monografia (Trabalho de Conclusão de Curso) - Centro de Psicologia Aplicada, Departamento de Psicologia, Faculdade de Ciências, Universidade Estadual Paulista, Bauru, 2001. Disponível em: <http://www.profla.com/artpsico53.htm>. Acesso em: 03 abr. 2011.

SCOCHI, C. G. S.; FERREIRA, F. Y.; GOES, F. S. N.; FUJINAGA, C. I.; FERECINI, G. M.; LEITE, A. M. Alimentação láctea e prevalência do aleitamento materno em prematuros durante internação em um hospital amigo da criança de Ribeirão Preto-SP, Brasil. Ciência e Cuidados em Saúde, [S.I.], v. 7, n. 2, p. 145-154, 2008.

TRINDADE, C. E. P.; LYRA, J. C. O recém-nascido pré-termo. In: ALVES FILHO, N. et al. Perinatologia básica. 3. ed. Rio de Janeiro: Guanabara Koogan, 2006. p. 406-416.

UNIVERSIDADE FEDERAL DE SÃO CARLOS - UFSCAR. 2011. Disponível em: http://www.ufscar.br. Acesso em: 03 maio 2012.

VAZ, F. A. C. Prematuridade: fatores etiológicos. Pediatria (São Paulo), São Paulo, v. 8, p. 169-171, 1986.

WEISS, M. C.; FUJINAGA, C. I. Prevalência de nascimentos baixo peso e prematuros na cidade de Irati-PR: implicações para a fonoaudiologia. Revista Salus, Guarapuava, v. 1, n. 2, p. 123-127, 2007.

WHO. The worldwide incidence of preterm birth: a systematic review of maternal mortality and morbidity. Bulletin of the World Health Organization, Geneva, v. 88, p. 31-38, 2010. 
WILLRICH, A.; AZEVEDO, C. C. F.; FERNANDES, J. O. Desenvolvimento motor na infância: influência dos fatores de risco e programas de intervenção. Rev. Neurociências, São Paulo, v. 17, n. 1, p. 51-56, 2009.

\section{Correspondência}

Luciana Pizzani - Rodovia Washington Luiz, KM 235 - Programa de Pós-Graduação em Educação Especial - Universidade Federal de São Carlos - São Carlos, São Paulo. CEP: 13560000.

E-mail: lupizzani@hotmail.com

Recebido em 04 de maio de 2012

Aprovado em 18 de junho de 2012 